\title{
$t$-LINKED OVERRINGS OF NOETHERIAN WEAKLY FACTORIAL DOMAINS
}

\author{
MARY B. MARTIN AND M. ZAFRULLAH
}

(Communicated by Louis J. Ratliff, Jr.)

\begin{abstract}
An integral domain $D$ is a WFD if each nonzero nonunit of $D$ is a product of primary elements of $D$. We show that each $t$-linked overring of a Noetherian WFD is again a WFD. This leads to the conclusion that the integral closure of a Noetherian WFD is a UFD.
\end{abstract}

An integral domain $D$ is called a weakly factorial domain (or a WFD for short) if each nonzero nonunit of $D$ is expressible as a product of primary elements of $D$. The aim of this note is to indicate how close WFD's are to UFD's, especially when they are Noetherian. For instance, as a consequence of one of the results, we show that the integral closure of a Noetherian WFD is a UFD. This improves on the following statement, which using a suggestion of David Anderson was proved in [1]: If $D$ is a one-dimensional Noetherian WFD then its integral closure $D^{\prime}$ is a PID. We also show that a flat overring of a WFD $D$ is a quotient ring of $D$.

Introduced in [1], WFD's were considered a somewhat weak notion but recently some interesting properties of these rings were discovered in [2]. These properties indicate that the study of WFD's may well be worthwhile. It was shown in [2] that the following properties are equivalent for an integral domain $D$ : (1) $D$ is a WFD; (2) Every convex directed subgroup of the group of divisibility $G(D)$ is a cardinal summand of $G(D) ;(3)$ For each $x$ in $D \backslash\{0\}$, if $P$ is a minimal prime over $x D$ then ht $P=1$ and $x D_{P} \cap D$ is principal; and (4) $D=\bigcap_{P \in X^{1}(D)} D_{P}$ is a locally finite intersection and every $t$-invertible $t$-ideal is principal. Here $X^{1}(D)$ denotes the set of all ht 1 primes of $D$.

Let $K$ be the quotient field of $D$ and call a ring $R$ with $D \subseteq R \subseteq K$ an overring of $D$. An overring $R$ of $D$ is said to be t-linked over $D$ if for any finitely generated ideal $A$ of $D, A^{-1}=D$ implies $(A R)^{-1}=R$ [3]. Now the integral closure of a Noetherian domain $D$ is $t$-linked over $D$ [3, Corollary 2.14] and a Krull domain that is also a WFD is a UFD [1, Theorem 15]. So if we show that every $t$-linked overring of a Noetherian WFD is again a WFD, then we are more than done. This is precisely what we intend to do. Before we embark on the stated course, we introduce the terminology that we shall use frequently. We call $x$ and $y$ in $D \quad v$-coprime if $x D \cap y D=x y D$ (or equivalently if

Received by the editors July 5, 1990 and, in revised form, December 12, 1990.

1980 Mathematics Subject Classification(1985 Revision). Primary 13A05, 13A17, 13B20, 13 F99. 
$\left.(x, y)_{v}=D\right)$. An integral domain $D$ is called a Mori domain if $D$ satisfies ACC on integral divisorial ideals. It is known that a locally finite intersection of Mori domains is again a Mori domain [6]. While every Noetherian domain is a Mori domain, a Mori domain may not be Noetherian. We call $D$ an infra-Krull domain, if $D=\bigcap_{P \in X^{1}(D)} D_{P}$ where the intersection is locally finite and for each $P$ in $X^{1}(D), D_{P}$ is a Noetherian domain. Obviously an infra-Krull domain is a Mori domain while a Mori domain need not be infra-Krull ... even if $D=\bigcap_{P \in X^{1}(D)} D_{P}$; there do exist one-dimensional Mori domains that are not Noetherian [3, Remark 2.21]. The reason for this nomenclature is obvious as $D$ is Krull if $D=\bigcap_{P \in X^{1}(D)} D_{P}$, where the intersection is of finite character and $D_{P}$ is a DVR for each $P$ in $X^{1}(D)$. We use star operations and related terminology freely. Readers in need of refreshing their memory can consult [4, $\S 32$ and 34]. Using the terminology, we can state our main result in the following most general form.

Theorem. Every t-linked overring of an infra-Krull WFD is and infra-Krull WFD.

To prove this theorem we need the following lemmas.

Lemma 1. Let $D$ be a Mori domain. Then every maximal t-ideal of $D$ is of height one if and only if $D=\bigcap_{P \in X^{1}(D)} D_{P}$. Consequently if $D$ is infra-Krull then every maximal $t$-ideal of $D$ is a height one prime ideal.

Proof. According to Querre [5], for every ideal $A$ of a Mori domain there is a finitely generated ideal $B \subseteq A$ such that $A_{v}=B_{v}$. So for any nonzero ideal $A, A_{v}=D$ implies that $A_{t}=D$. Now let $P$ be a maximal $t$-ideal of $D$. Then $P=P_{v}=B_{v}$ for some finitely generated $B \subseteq P$. It is sufficient to show that $P$ is contained in a member of $X^{1}(D)$. For this we assume that $P \nsubseteq Q$ for all $Q$ in $X^{1}(D)$ and let * be the *-operation induced on $D$ by $\left\{D_{P}\right\}$ then $P^{*}=\bigcap_{Q \in X^{1}(D)} P D_{Q}=D$. But then $P=P_{v}=\left(P^{*}\right)_{v}=D$, a contradiction. Conversely if every maximal $t$-ideal of $D$ is of height one then as $D=\bigcap D_{P}$ where $P$ ranges over maximal $t$-ideals and as each $P \in X^{1}(D)$ is a $t$-ideal we have the conclusion.

Lemma 2. Each t-linked overring of an infra-Krull domain is an infra-Krull domain.

Proof. Clearly, according to Lemma 1, a Mori domain is infra-Krull if and only if for each prime $t$-ideal $P$ of $D, D_{P}$ is a one-dimensional Noetherian domain. Now let $R$ be a $t$-linked overring of $D$. Then according to [3, Proposition 2.13] and by Lemma $1, R=\bigcap_{P \in X^{1}(D)} R_{(D \backslash P)}$. But each $R_{D \backslash P}$ is an overring of $D_{P}$ and hence is a semilocal one-dimensional Noetherian domain or the field of fractions of $D$. Further, as every nonzero nonunit $x$ of $D$ is a unit in almost all $R_{D \backslash P}$, the intersection $R=\bigcap_{P \in X^{\prime}(D)} R_{(D \backslash P)}$ is of finite character. Whence $R$ is a Mori domain. Now let $\underline{P}$ be a prime $t$-ideal in $R$. Then as $R$ is Mori, $(\underline{P})_{v}=\underline{P}$. So by the argument of Lemma $1, \underline{P} R_{(D \backslash P)} \neq R_{D \backslash P}$ for some $P$. But then $R_{\underline{P}}$ must be an overring of $R_{D \backslash P}$ and hence one-dimensional Noetherian.

Corollary 3. Let $D$ be a Noetherian domain such that every grade one prime of $D$ is of height one. Then every t-linked overring of $D$ is infra-Krull.

Now for the next leg of the journey we prove the following Lemma. 
Lemma 4. Let $D$ be a $W F D$, and let $R$ be t-linked over $D$. Let $p_{1}$ and $p_{2}$ be two primary elements of $D$ with $\sqrt{\left(p_{1}\right)} \neq \sqrt{\left(p_{2}\right)}$, and let $p_{1} / q_{1}, p_{2} / q_{2}$ be elements of $R$ such that for each $i, \sqrt{\left(q_{i}\right)}=\sqrt{\left(p_{i}\right)}$ or $q_{i}=1$. Then $\left(p_{1} / q_{1}, p_{2} / q_{2}\right)_{v}=R$.

Proof. Because $R$ is $t$-linked over $D$, we have by [3, Proposition 2.13], $R=$ $\bigcap_{P \in X^{1}(D)} R_{(D \backslash P)}$. Moreover, $p_{1}$ and $p_{2}$ are $v$-coprime in $D$ (since $\sqrt{\left(p_{1}\right)} \neq$ $\left.\sqrt{\left(p_{2}\right)}\right)$. So if $P$ is in $X^{1}(D)$ and $p_{1} \in P$, then $p_{2}, q_{2}$ are in $D \backslash P$ and so are units of $R_{(D \backslash P)}$. Consequently $\left(p_{1} / q_{1}, p_{2} / q_{2}\right) R_{(D \backslash P)}=R_{(D \backslash P)}$. The same holds for $p_{2} \in P$. If ${ }^{*}$ is the star operation induced by $\left\{R_{(D \backslash P)}\right\}_{P \in X^{1}(D)}$ on $R$ then $\left(p_{1} / q_{1}, p_{2} / q_{2}\right)^{*}=\bigcap_{P \in X^{1}(D)}\left(p_{1} / q_{1}, p_{2} / q_{2}\right) R_{(D \backslash P)}=\bigcap_{P \in X^{1}(D)} R_{(D \backslash P)}=R$ and consequently $\left(p_{1} / q_{1}, p_{2} / q_{2}\right)_{v}=R$.

With this preparation we are ready to prove the main theorem.

Proof of the main theorem. Let $D$ be infra-Krull and let $R$ be a $t$-linked overring of $D$. Then $R=\bigcap_{P \in X^{1}(D)} R_{(D \backslash P)}$ is infra-Krull, by Lemma 2. So every minimal prime of a principal ideal of $R$ is a height one prime of $R$, by Lemma 1. According to [2] all we have to show is that for each $0 \neq x \in \underline{P} \in X^{1}(R)$, $x R_{\underline{P}} \cap R$ is principal. Because $R$ is $t$-linked over $D$ and because every maximal $t$-ideal of $D$ is of height one, for each $\underline{P}$ in $X^{1}(R), \underline{P} \cap D$ is a height one prime $t$-ideal [3, Proposition 2.1]. Let $\underline{P} \cap D=P$. Then $R_{\underline{P}}$ is an overring of $D_{P}$ and so $x R_{\underline{P}}=(p / q) R_{\underline{P}}$, where $\sqrt{(p)}=P, q=1$, or $\sqrt{(q)}=P$ and $p / q \in R$. Now $x R_{\underline{P}} \cap R=x R_{\underline{P}} \cap R_{(D \backslash P)} \cap R$. As $R_{(D \backslash P)}$ is an overring of $D_{P}$, which is one-dimensional Noetherian, $R_{(D \backslash P)}$ is one-dimensional semilocal. So, according to [1] $R_{(D \backslash P)}$ is a WFD. Therefore $x R_{\underline{P}} \cap R_{(D \backslash P)}=h R_{(D \backslash P)}$, where $h$ is in $R$ and $\sqrt{h R_{(D \backslash P)}}=\underline{P} R_{(D \backslash P)}$. Also, $h=p_{2} / q_{2}$ such that $\sqrt{\left(p_{2}\right)}=P$ and $q_{2}=1$ or $\sqrt{\left(q_{2}\right)}=P$ in $D$. Now let $\overline{D \backslash P}$ denote the saturation of $D \backslash P$ in $R$. Then $k$ in $\overline{D \backslash P}$ implies that $k=\alpha_{1} \alpha_{2} \cdots \alpha_{r} / \beta_{1} \beta_{2} \cdots \beta_{s}$ where $\alpha_{i}, \beta_{j}$ are primary elements of $D$ coming from $D \backslash P$. But then by Lemma 4 (and by the fact that if $(a, b)_{v}=(a, c)_{v}=D$ then $\left.(a, b c)_{v}=D\right),(h, k)_{v}=R$. Now $h R_{D \backslash P} \cap R=\{x \in R|h| x s$ for some $s$ in $\overline{D \backslash P}\}$. But if $h \mid x s$ and $(h, s)_{v}=R$, then $h$ divides $x$ [7, Lemma 1.1]. So $h R_{(D \backslash P)} \cap R=h R$. That is, $x R_{\underline{P}} \cap R$ is principal.

Now the promised result.

Corollary 5. The integral closure of a Noetherian WFD, D is a UFD.

Proof. As we have already noted the integral closure $D^{\prime}$ of $D$ is a WFD because $D^{\prime}$ is $t$-linked over $D$ [3] and, as $D^{\prime}$ is a Krull domain, we conclude that $D^{\prime}$ is a Krull WFD. But a Krull WFD is a UFD [1].

In general, overrings of a one-dimensional quasilocal domain cannot be semiquasilocal and/or one dimensional. So the above results cannot be stated for general WFD's. Yet there is another breed of $t$-linked overrings for which a semblance of the above results follows through.

Let $D$ be a WFD and let $\varnothing \neq Y \subseteq X^{1}(D)$. Then $R=\bigcap_{P \in Y} D_{P}$ can be called a subintersection.

Proposition 8. Let $D$ be a $W F D$. Then every subintersection of $D$ is a quotient ring of $D$. 
Proof. Let $R=\bigcap D_{P}$ where $P$ ranges over $Y \subseteq X^{1}(D)$, and let $S=\{s \in D \mid s$ is a unit in $R$. Clearly $R \supseteq D_{S}$.

Now let $x$ be a nonzero nonunit of $R$, then $x=\alpha / \beta$ where $\alpha, \beta \in D \backslash\{0\}$. So we can write $x$ as $p_{1} p_{2} \cdots p_{r} / q_{1} q_{2} \cdots q_{s}$ where $p_{i}, q_{j}$ are primary, and we can assume that the $p_{i}$ are all mutually $v$-coprime and so are the $q_{j}$. Now as $x$ is a nonunit in $R$, there is a $\underline{P}$ in $X^{1}(R)$ such that $x$ is in $\underline{P}$. So $x D_{P} \neq D_{P}$ for some $P$ in $Y$. But then there is a $p_{i}$ in the numerator of $x$, such that $\sqrt{\left(p_{i}\right)}=P$. We claim that $\left(p_{i}, q_{j}\right)_{v} \neq D$ implies that $q_{j} \mid p_{i}$ in $R$. For, then $q_{j} \in P$ and so belongs to no other height 1 prime in $Y$ and $p_{i} / q_{j} D_{P}=x D_{P}$ requires that $q_{j}$ divides $p_{i}$. Thus we can assume that $\left(p_{i}, q_{j}\right)_{v}=D$ for all $q_{j}$ in the denominator. Repeating this process for all $\underline{P}$ in $X^{1}(R)$, we conclude that $(\alpha, \beta)_{v}=D$. Consequently $\alpha$ and $\beta$ share no height 1 prime of $D$ and hence of $R$. But then $\beta$ is in no member of $X^{1}(R)$ and so is a unit in $R$. Consequently $x=\alpha / \beta$ where $\beta \in S$ and so $R=D_{S}$.

Corollary 9. Every flat overring of a WFD $D$ is a quotient ring of $D$.

Proof. Obviously a flat overring of a WFD is a subintersection.

\section{ACKNOWLEDGMENTS}

The material in this note has benefited from the encouraging criticism of Professors D. E. Dobbs and D. F. Anderson. We are thankful also to the referee for pointing out several typographical errors and recommending some useful clarifications.

\section{REFERENCES}

1. D. D. Anderson and L. A. Mahaney, On primary factorizations, J. Pure Appl. Algebra 54 (1988), 141-154.

2. D. D. Anderson and M. Zafrullah, Weakly factorial domains and groups of divisibility, Proc. Amer. Math. Soc. 109 (1990), 907-913.

3. D. Dobbs, E. Houston, T. Lucas, and M. Zafrullah, t-linked overrings and Prufer $v$ multiplication domains, Comm. Algebra 17 (1989), 2835-2852.

4. R. W. Gilmer, Multiplicative ideal theory, Marcel Dekker, New York, 1972.

5. J. Querre, Sur une propriete des anneaux de Krull, Bull. Sci. Math. (2) 95 (1971), 341-354.

6. N. Raillard, Sur les anneaux de Mori, thesis, Paris VI, 1976.

7. M. Zafrullah, A general theory of almost factoriality, Manuscripta Math. 51 (1985), 29-62.

Department of Mathematics, Winthrop College, Rock Hill, South Carolina 29733 